\title{
FITORREMEDIAÇÃO DE SOLOS CONTAMINADOS COM ARSÊNIO (AS) UTILIZANDO BRAQUIÁRIA
}

\author{
Phytoremediation of arsenic-contaminated soils using Brachiaria grass
}

\begin{abstract}
Alessandra Silveira Antunes Araújoํㅜ, Luiz Roberto Guimarães Guilherme², Guilherme Lopes², Mari Lúcia Campos ${ }^{3}$
RESUMO

Conduziu-se este trabalho, com o objetivo de avaliar o potencial fitoextrator de arsênio (As) da Brachiaria decumbens Stapf., cultivada em casa de vegetação, utilizando-se seis solos: Latossolo Amarelo Distrófico (LAd), Latossolo Vermelho Distrófico (LVd), Neossolo Flúvico (RU), Neossolo Quartzarênico (RQ), Gleissolo Háplico (GX) e Gleissolo Melânico (GM). O As foi adicionado aos solos na forma de arsenato de sódio heptahidratado nas doses $0,25,50,200$ e $800 \mathrm{mg} \mathrm{kg}^{-1}$. As plantas foram coletadas 55 dias após a semeadura, separadas em parte aérea e raiz e, após secagem do material em estufa, o mesmo foi digerido segundo o método 3051 da USEPA, sendo o teor de As determinado por espectrofotometria de absorção atômica (EEA) com forno de grafite. Observou-se redução no desenvolvimento da braquiária de forma diferenciada nas seis classes de solo. Houve baixa translocação do As, da raiz para a parte aérea, sendo essa espécie considerada tolerante ao elemento e não hiperacumuladora, podendo ser utilizada em programas de revegetação de áreas contaminadas pelo elemento.
\end{abstract}

Termos para indexação: Fitoextração, Brachiaria decumbens, contaminação ambiental.

\section{ABSTRACT}

This work evaluated the phytoextraction potential of arsenic (As) by Brachiaria decumbens growing under greenhouse conditions in six soils: a Dystrophic Yellow Latosol (LAd), a Dystrophic Red Latosol (LVd), a Fluvic Neosol (RU), a Quartzenic Neosol (RQ), a Haplic Gleysol (GX), and a Melanic Gleysol (GM). Arsenic was added to the soils as sodium arsenate heptahydrate $\left(\mathrm{Na}_{2} \mathrm{HAsO}_{4} .7 \mathrm{H}_{2} \mathrm{O}\right)$ at the following doses: $0,25,50,200$, and $800 \mathrm{mg} \mathrm{kg}^{-1}$. The plants were collected 55 days after planting for determination of shoot and root dry matter production. Arsenic shoot and root contents were evaluated after digestion according to the USEPA 3051A method, following analysis by graphite furnace atomic absorption spectroscopy. Plant development was differently affected by As in the six different soil classes. There was low translocation of As and consequently most of the As was detected in the roots of Brachiaria decumbens. Therefore, this species could be considered tolerant but not an accumulator of arsenic, thus being indicated for revegetation programs in As-polluted soils and sediments.

Index terms: Phytoextraction, Brachiaria decumbens, environmental contamination.

(Recebido em 16 de abril de 2009 e aprovado em 30 de abril de 2010)

\section{INTRODUÇÃO}

O arsênio (As), desde 1997, vem sendo considerado o elemento mais tóxico à saúde humana, segundo a Lista de Prioridade de Substâncias Perigosas da ATSDR (Agency for Toxic Substances \& Disease Registry, 2006). Em muitas partes do mundo, a contaminação pelo As tem causado problemas ambientais e de saúde (Gonzaga et al., 2006). As principais vias de exposição são a ingestão de água, alimentos e solo e, em alguns casos, a inalação de arsênio do ar (Barra et al., 2000; Basu et al., 2001; Baird, 2002; Fitz \& Wenzel, 2002). Os problemas gerados à saúde pelo elemento dependem de fatores como quantidade ingerida, dose, frequência e tempo de absorção, resultando em efeitos tóxicos, agudos ou crônicos (Borba et al., 2004).
Os processos de contaminação das águas e dos solos podem ser tanto naturais como antrópicos e o grau de risco de contaminação está associado às formas de As que estão biologicamente disponíveis para absorção, ou biodisponíveis para humanos (Rodriguez et al., 2003). A mobilidade e a biodisponibilidade de arsênio no solo são controladas, principalmente, por reações de adsorção e de dessorção (Smith et al., 2002), sendo que a adsorção, a dessorção e a concentração de As na solução do solo são controladas pelos seus atributos físicos, químicos e biológicos (Campos et al., 2006).

A fitorremediação consiste em uma técnica relativamente nova para descontaminação ambiental (Lindblom et al., 2006), utilizando plantas e microrganismos a elas associados para extrair, degradar ou estabilizar o contaminante do solo, sedimentos e corpos d'água (Terry \&

\footnotetext{
1Universidade Federal de Lavras/UFLA - Cx. P. 37 - 37200-000 - Lavras, MG - alessandra_santunes@yahoo.com.br 2Universidade Federal de Lavras/UFLA - Departamento de Ciência do Solo/DCS - Lavras, MG

3Universidade do Estado de Santa Catarina/UDESC - Departamento de Solos - Lages, SC
} 
Banuelos, 2000, citados por Cai et al., 2004). Ao se escolherem espécies de plantas para serem utilizadas em programas de fitorremediação, especialmente fitoextração, devem-se considerar fatores do solo e da planta, como desenvolvimento da planta, nível de contaminação, profundidade do sistema radicular (Gonzaga et al., 2006), produção de biomassa, os fatores de bioacumulação e de transferência e a capacidade em hiperacumular o elemento (Fayiga et al., 2004).

Há, no Brasil, registros de ocorrência de contaminação de águas (até $0,36 \mathrm{mg} \mathrm{L}^{-1}$ ), solos (até $860 \mathrm{mg} \mathrm{kg}^{-1}$ ) e sedimentos (até $3.200 \mathrm{mg} \mathrm{kg}^{-1}$ ) por As nas proximidades de áreas industriais ou de mineração (Magalhães \& Pfeiffer, 1995; Matschullat et al., 2000; Magalhães et al., 2001). A utilização de plantas na revegetação de ambientes contaminados com As tem sido bastante indicada, uma vez que apresenta menor custo e menor impacto ao meio ambiente.

\section{MATERIAL E MÉTODOS}

O presente estudo foi conduzido em casa de vegetação do Departamento de Ciência do Solo da Universidade Federal de Lavras. Foram utilizadas seis classes de solos: Neossolo Flúvico (RU), Gleissolo Háplico (GX), Gleissolo Melânico (GM) e Latossolo Vermelho Distrófico (LVd), coletados no município de Lavras-MG; Neossolo Quartzarênico (RQ), coletado no município de Itutinga-MG; e Latossolo Amarelo Distrófico (LAd), coletado no município de Rosário-MG (Tabelas 1 e 2).

Tabela 1 - Análise granulométrica e concentrações de alumínio e ferro, por ataque sulfúrico, dos solos utilizados para o cultivo da Brachiaria decumbens.

\begin{tabular}{|c|c|c|c|c|c|}
\hline \multirow{3}{*}{ Solo } & \multicolumn{3}{|c|}{ Análise granulométrica } & \multicolumn{2}{|c|}{ Ataque sulfúrico } \\
\hline & Argila & Areia & Silte & $\mathrm{Al}_{2} \mathrm{O}_{3}$ & $\mathrm{Fe}_{2} \mathrm{O}_{3}$ \\
\hline & \multicolumn{5}{|c|}{---------------------------- g kg $^{-1}$--------------------------- } \\
\hline LAd & 220 & 680 & 100 & 71,6 & 25,5 \\
\hline LVd & 720 & 180 & 100 & 270 & 125 \\
\hline RU & 310 & 420 & 270 & 221,1 & 74,5 \\
\hline GX & 160 & 500 & 340 & 112,2 & 13,0 \\
\hline GM & 370 & 240 & 390 & 175,5 & 13,3 \\
\hline RQ & 60 & 920 & 20 & 35,8 & 11 \\
\hline
\end{tabular}

Fonte: Fernandes et al, 2000; Souza et al, 2006.

Tabela 2 - Principais atributos químicos dos solos utilizados para o cultivo da Brachiaria decumbens.

\begin{tabular}{|c|c|c|c|c|c|c|c|c|c|c|c|c|c|c|}
\hline \multirow{3}{*}{ Solos } & \multicolumn{14}{|c|}{ Atributos químicos ${ }^{(*)}$} \\
\hline & \multirow{2}{*}{$\mathrm{pH}$} & $\mathrm{P}$ & $\mathrm{K}$ & $\mathrm{Ca}^{2+}$ & $\mathrm{Mg}^{2+}$ & $\mathrm{Al}^{3+}$ & $\mathrm{H}+\mathrm{Al}$ & SB & $(\mathrm{t})$ & $(\mathrm{T})$ & $\mathrm{V}$ & $\mathrm{m}$ & MO & P-rem \\
\hline & & \multicolumn{3}{|c|}{$\mathrm{mg} \mathrm{dm}{ }^{-3}$} & \multicolumn{6}{|c|}{ 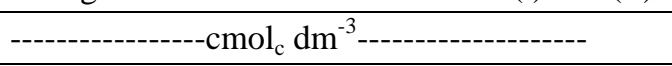 } & \multicolumn{2}{|c|}{$----\%----$} & dag $\mathrm{kg}^{-1}$ & $\mathrm{mg} \mathrm{L}^{-1}$ \\
\hline RQ & 4,6 & 10,8 & 30 & 0,7 & 0,2 & 1,0 & 3,6 & 1,0 & 2,0 & 4,6 & 21,4 & 51 & 1,4 & 37,7 \\
\hline RU & 4,8 & 2,3 & 97 & 1,1 & 0,8 & 0,9 & 5,0 & 2,2 & 3,1 & 7,2 & 30,1 & 30 & 1,9 & 16,0 \\
\hline LAd & 4,5 & 2,0 & 84 & 0,7 & 0,7 & 0,8 & 4,0 & 1,6 & 2,4 & 5,6 & 28,7 & 33 & 2,5 & 38,7 \\
\hline $\mathrm{LVd}$ & 4,4 & 1,7 & 48 & 0,8 & 0,2 & 1,6 & 12,3 & 1,1 & 2,7 & 13,4 & 8,3 & 59 & 4,6 & 11,5 \\
\hline GX & 4,2 & 6,8 & 45 & 0,5 & 0,2 & 2,0 & 7,9 & 0,8 & 2,8 & 8,7 & 9,4 & 71 & 2,9 & 33,8 \\
\hline GM & 4,4 & 6,5 & 58 & 1,3 & 0,7 & 2,1 & 11,0 & 2,2 & 4,3 & 13,2 & 16,3 & 49 & 6,3 & 10,2 \\
\hline
\end{tabular}

${ }^{(*)}$ Atributos químicos: $\mathrm{pH}$ - pH em água (relação 1:2,5); P (fósforo) e $\mathrm{K}$ (potássio) - extrator Mehlich 1; Ca (cálcio), Mg (magnésio), $\mathrm{Al}$ (alumínio) - extrator $\mathrm{KCl} 1 \mathrm{~mol} \mathrm{~L}^{-1} ; \mathrm{H}+\mathrm{Al}$ (hidrogênio + alumínio) - extrator SMP; SB (soma de bases trocáveis); $\mathrm{t}$ (capacidade de troca catiônica efetiva); $\mathrm{T}$ (capacidade de troca catiônica a $\mathrm{pH} 7,0$ ); V (saturação por bases); $\mathrm{m}$ (saturação por alumínio); $\mathrm{MO}$ (matéria orgânica) - oxidação: $\mathrm{Na}_{2} \mathrm{Cr}_{2} \mathrm{O}_{7} 0,67 \mathrm{~mol} \mathrm{~L}^{-1}+\mathrm{H}_{2} \mathrm{SO}_{4} 5 \mathrm{~mol} \mathrm{~L}-1$ e P-rem (fósforo remanescente) - extrator CaCl $210 \mathrm{mmol} \mathrm{L}^{-1}$. Atributos: pH, P, K, Ca, Mg, Al e H + Al foram feitos conforme Empresa Brasileira de Pesquisa Agropecuária - Embrapa (1997), MO segundo Vettori (1969) e P-rem segundo Ribeiro et al. (1999). 
Os solos utilizados para o cultivo da Brachiaria decumbens Stapf. apresentam diferentes texturas e teores de matéria orgânica e foram incubados com arsênio na forma de arsenato de sódio heptahidratado $\left(\mathrm{Na}_{2} \mathrm{HAsO}_{4} .7 \mathrm{H}_{2} \mathrm{O}\right)$ nas doses 0, 25, 50, 200 e $800 \mathrm{mg} \mathrm{kg}^{-1}$ de Âs. Antes de serem incubados, esses solos tiveram seus teores naturais de As analisados utilizando o método USEPA 3051 A (Tabela 3). O período de incubação dos solos foi de 6 meses, e estes foram mantidos próximos da capacidade de campo e em ambiente arejado. Para o cultivo da braquiária, corrigiu-se o $\mathrm{pH}$ para aproximadamente 5,5, mediante a adição de hidróxido de cálcio e hidróxido de magnésio, na proporção de 3:1, respectivamente. Após a adição dos hidróxidos, os solos foram mantidos com umidade próxima à capacidade de campo. Durante esse período, realizou-se um acompanhamento do $\mathrm{pH}$, por meio de leituras que foram efetuadas a cada 3 dias. $\mathrm{O}$ pH foi considerado estável quando os valores variaram menos de 0,3 unidade da leitura anterior. A estabilidade foi conseguida após 18 dias da adição dos hidróxidos, ocasião em que os solos apresentavam valores de $\mathrm{pH}$ próximos de 5,5.

Tabela 3 - Concentrações naturais de As, extraídas pelo método USEPA 3051 e determinadas por EEA com forno de grafite, nos solos utilizados para o cultivo da Brachiaria decumbens.

\begin{tabular}{cc}
\hline Solo & Teor de As $\left(\mathrm{mg} \mathrm{kg}^{-1}\right)$ \\
\hline Lad & $1,09 \pm 0,27$ \\
LVd & $9,26 \pm 1,02$ \\
RU & $0,43 \pm 0,37$ \\
RQ & $0,33 \pm 0,30$ \\
GX & $0,14 \pm 0,06$ \\
GM & $0,86 \pm 0,26$ \\
\hline
\end{tabular}

O delineamento experimental adotado foi inteiramente casualizado (DIC), com 3 repetições. Os tratamentos foram constituídos pela combinação de 6 classes de solos (RU, GX, GM, LVd, RQ, LAd) e 5 doses de As $\left(0,25,50,200,800 \mathrm{mg}\right.$ de $\left.\mathrm{As} \mathrm{kg}^{-1}\right)$, totalizando 90 parcelas.

Visando a obter uma germinação mais homogênea da braquiária, foi efetuada a quebra da dormência das sementes utilizando ácido sulfúrico concentrado (18 $\left.\mathrm{mol} \mathrm{L}^{-1}\right)$ durante, aproximadamente, 2 minutos e, em seguida, lavagem com água corrente e secagem à sombra para posterior semeadura (Brasil, 1992). Adicionaram-se $10 \mathrm{~cm}^{3}$ de semente por vaso e, após a germinação, foram realizados desbastes, restando, em média, 10 plantas por vaso. Diariamente, os vasos foram irrigados com água deionizada, com o intuito de manter a sua capacidade de campo e com solução $0,1 \mathrm{mmol} \mathrm{L}^{-1} \mathrm{de}$ sulfato de cálcio dihidratado durante a primeira semana, após a semeadura, e a cada 2 dias no decorrer do experimento, visando ao maior desenvolvimento de raízes.

Foram realizadas adubações, em que as doses de nutrientes aplicados, em $\mathrm{mg} \mathrm{dm}^{-3}$, foram as seguintes: 300 de nitrogênio, 200 de fósforo, 300 de potássio, 75 de cálcio, 30 de magnésio, 50 de enxofre, 0,5 de boro, 1,5 de cobre, 5 de ferro, 10 de manganês e 0,1 de molibdênio. Com exceção do nitrogênio e do potássio, que foram parcelados em três aplicações, os outros nutrientes foram aplicados em uma única vez.

As plantas foram coletadas 55 dias após a emergência, separadas em parte aérea e raiz, lavadas com água deionizada e secas em estufa com circulação forçada de ar, a uma temperatura de aproximadamente $65^{\circ} \mathrm{C}$, por cerca de $72 \mathrm{~h}$. Após a secagem, o material foi pesado para avaliação da produção de matéria seca de raiz (MSR) e parte aérea (MSPA) e, em seguida, triturado. O material triturado, posteriormente digerido segundo método 3051 da USEPA, foi analisado por EEA com forno de grafite. $O$ limite de detecção qualitativo do método USEPA 3051, corresponde a $0,34 \mathrm{mg} \mathrm{kg}^{-1}$ e foi calculado utilizando a equação a seguir:

\section{$\mathrm{LDQ}=\mathrm{Fd} *(\mathrm{M} \pm \mathrm{k} * \mathrm{~s})$}

em que: LQD é o limite de detecção qualitativo do método analítico, Fd é o fator de diluição das amostras, M é a média das provas em branco, $\mathrm{k}$ é o coeficiente de Kaiser que, possui valor fixo igual a 3 (Skoog et al., 2002), e s é o desvio padrão das provas em branco.

Para avaliar o potencial da braquiária na extração de As e identificá-la como uma espécie tolerante ou hiperacumuladora de As, adotaram-se os valores referentes ao fator de bioacumulação (FA) (McGrath \& Zao, 2003, citados por Caille et al., 2005) e ao índice de translocação (IT) (Abichequer \& Bohnen, 1998) do elemento na planta, conforme descrito a seguir:

FA $=\frac{\text { Concentração do elemento na parte aérea }}{\text { Teor do elemento no solo }}$

IT $=\frac{\text { Acúmulo do elemento na parte aérea }}{\text { Acúmulo do elemento na planta }} \times 100$ 
Foram realizadas análises qualitativas para avaliar o efeito das diferentes doses de As, aplicadas aos solos utilizados no cultivo da braquiária. Para verificar o efeito dos tratamentos sobre o desenvolvimento da braquiária e o teor de As na planta, foram calculadas médias e desvios padrões e realizadas comparações entre os resultados obtidos com as doses de As aplicadas e as propriedades de cada solo utilizado.

\section{RESULTADOS E DISCUSSÃO}

Em quase todos os solos aos quais foram aplicadas as doses de 200 e $800 \mathrm{mg} \mathrm{kg}^{-1}$ de As ocorreram menores produções de matéria seca de raiz (MSR) (Figura 1), sendo que não houve produção no LAd, no RQ e no GX na dose de $800 \mathrm{mg} \mathrm{kg}^{-1}$. A produção de MSR no RQ não ocorreu com a dose de $200 \mathrm{mg} \mathrm{kg}^{-1}$. Tal fato pode estar associado aos menores teores de argila, $\mathrm{Fe}_{2} \mathrm{O}_{3}$ e $\mathrm{Al}_{2} \mathrm{O}_{3}$ presentes nesse solo; ou seja, havia mais As disponível para ser absorvido pelas plantas. Nas doses de 0 , 25 e $50 \mathrm{mg} \mathrm{kg}^{-1}$ de As, em todas as classes de solo, não se observa muita variação na produção de MSR, o que pode estar relacionado ao fato de que tais concentrações se enquadram na faixa considerada normal para solos de acordo com Mandal \& Suzuki (2002), segundo os quais a concentração varia de $<0,1-97 \mathrm{mg} \mathrm{kg}^{-1}$. As produções de MSR dos solos LVd e RU, na dose equivalente a $200 \mathrm{mg} \mathrm{kg}^{-1}$ de As, foram semelhantes aos valores obtidos nas doses 0,25 e $50 \mathrm{mg} \mathrm{kg}^{-1}$ de As nas respectivas classes de solo.

A produção de MSR nas doses 200 e $800 \mathrm{mg} \mathrm{kg}^{-1}$ segue a seguinte ordem crescente: $\mathrm{RQ}<\mathrm{GX}<\mathrm{LAd}<\mathrm{GM}<$ $\mathrm{RU}<\mathrm{LVd}$. Dessa forma, observa-se a relação existente entre o teor de argila e a disponibilidade de As para as plantas, uma vez que a disponibilidade de As está altamente relacionada com as propriedades dos solos, como o teor de argila, óxidos de ferro e alumínio, que garantem adsorção específica e redução na mobilidade e na dessorção do As (Goh \& Lim, 2004). Logo, os solos RU e LVd, em razão de seus maiores teores de argila, $\mathrm{Fe}_{2} \mathrm{O}_{3}$ e $\mathrm{Al}_{2} \mathrm{O}_{3}$, podem aumentar a adsorção de arsênio e, consequentemente, torná-lo indisponível para as plantas.

A produção de matéria seca de parte aérea (MSPA) (Figura 2) respondeu aos tratamentos de forma semelhante ao que foi reportado anteriormente para a MSR. Logo, nas doses com 200 e $800 \mathrm{mg} \mathrm{kg}^{-1}$ de As, houve redução na produtividade, exceto para o LVd e RU na dose de $200 \mathrm{mg} \mathrm{kg}^{-1}$, cujos valores médios foram próximos aos obtidos nas doses 0,25 e 50. No RQ, a redução na produção de MSPA ocorreu na dose de $50 \mathrm{mg} \mathrm{kg}^{-1}$, e não com $200 \mathrm{mg} \mathrm{kg}^{-1}$ conforme foi observado para a produção de MSR. A produção de MSPA nas doses 200 e $800 \mathrm{mg} \mathrm{kg}^{-1}$ de As obedece à mesma sequência obtida para a produção de MSR.

Os teores médios de As na MSR se relacionaram com os teores de argila, $\mathrm{Fe}_{2} \mathrm{O}_{3}$ e $\mathrm{Al}_{2} \mathrm{O}_{3}$ presentes nos solos utilizados; ou seja, quanto maior o teor de argila e de óxidos de ferro e alumínio no solo, menor o teor de As na planta, em virtude dos processos de adsorção. Logo, a braquiária cultivada no solo RQ apresentou os maiores teores do elemento, e a cultivada no solo $\mathrm{LVd}$, os menores teores. A mesma correlação não foi verificada para a MSPA, pois os maiores teores de As foram obtidos no solo LAd e os menores, no solo RU, nas doses 0,25 e $50 \mathrm{mg} \mathrm{kg}^{-1}$ e no solo GM, nas doses 200 e $800 \mathrm{mg} \mathrm{kg}^{-1}$. Observa-se, também, que as variações nas concentrações médias de As na MSR e MSPA aumentaram expressivamente a partir da dose de $50 \mathrm{mg} \mathrm{kg}^{-1}$ em suas respectivas classes de solo (Figuras 3 e 4).

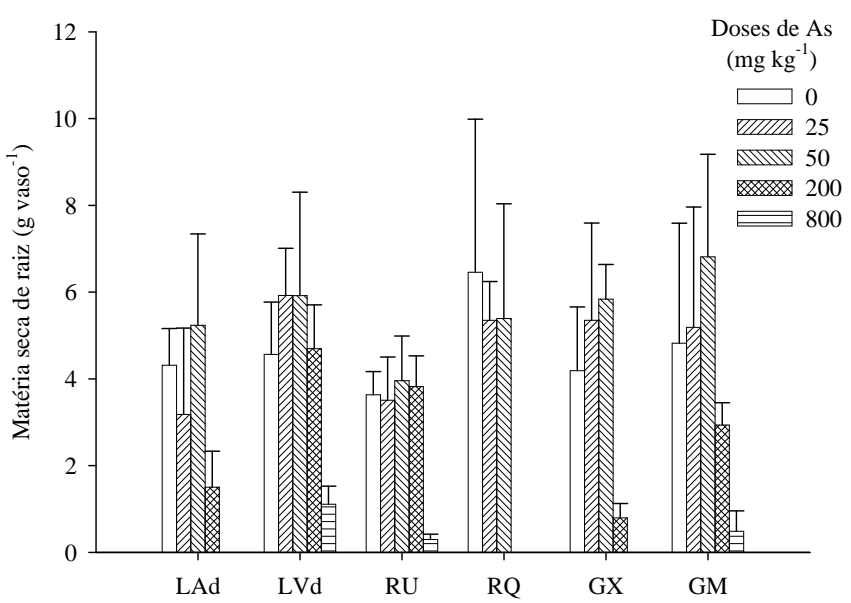

Figura 1 - Valores médios da produção de matéria seca de raiz da Brachiaria decumbens cultivada em solos contaminados artificialmente com As. 


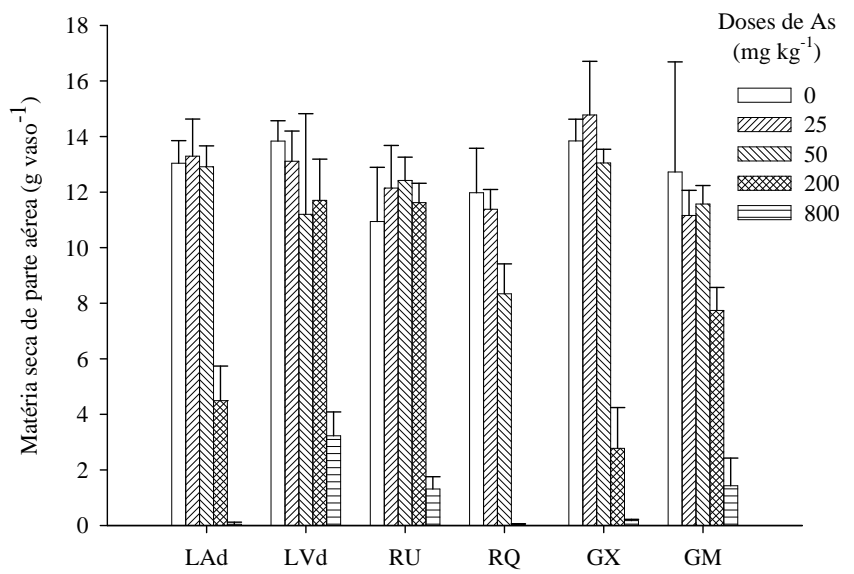

Figura 2 - Valores médios da produção de matéria seca de parte aérea da Brachiaria decumbens cultivada em solos contaminados artificialmente com As.

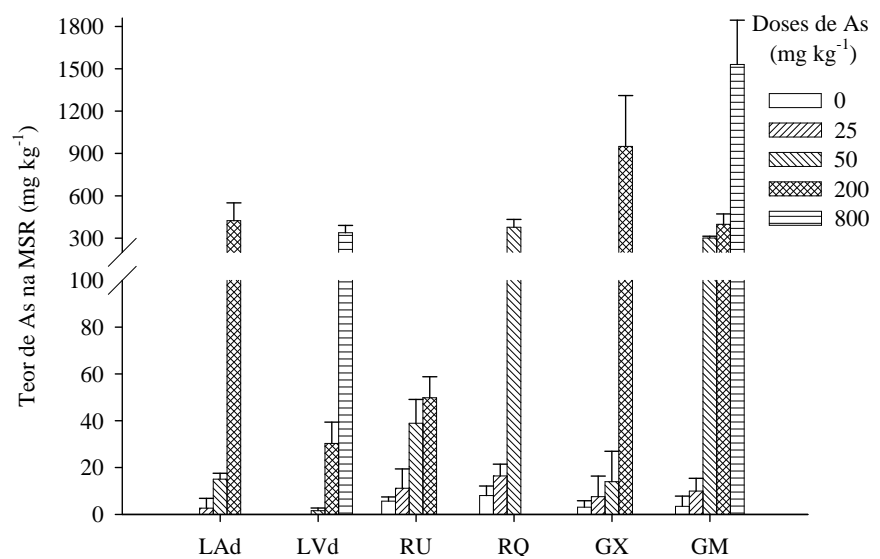

Figura 3 - Concentração média de As $\left(\mathrm{mg} \mathrm{kg}^{-1}\right)$ na matéria seca de raiz da Brachiaria decumbens cultivada em solos contaminados artificialmente com As.

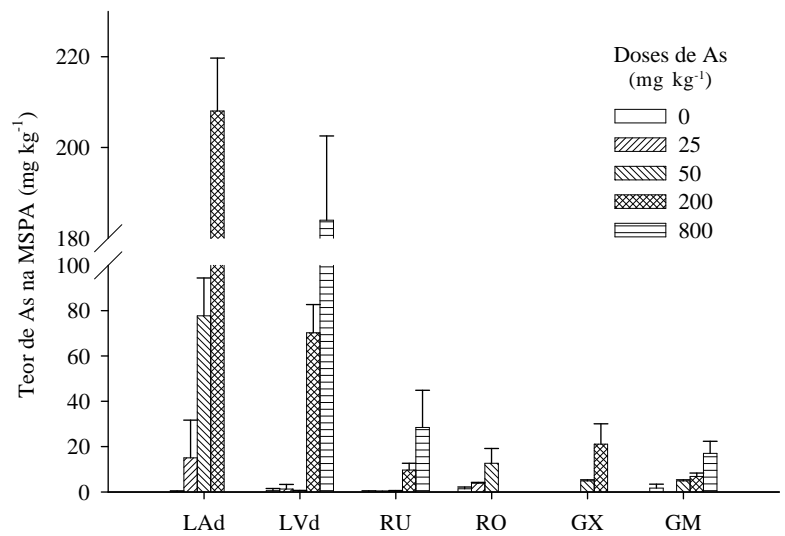

Figura 4 - Concentração média de $\mathrm{As}\left(\mathrm{mg} \mathrm{kg}^{-1}\right)$ na matéria seca de parte aérea da Brachiaria decumbens cultivada em solos contaminados artificialmente com As.

Ciênc. agrotec., Lavras, v. 35, n. 1, p. 84-91, jan./fev. 2011 
De acordo com Fitz \& Wenzel (2002), plantas tolerantes tendem a restringir a translocação de As do solo para a planta e da raiz para a parte aérea, enquanto as hiperacumuladoras absorvem e translocam ativamente o arsênio.

Plantas tolerantes possuem fator de bioacumulação menor que um. Um bom exemplo para tal afirmação é a comparação entre a planta tolerante Agrostis catellana Boiss. \& Reut. crescendo em solo que continha $17000 \mathrm{mg} \mathrm{kg}^{-1}$ de As (Koe \& Jaques, 1993) e a hiperacumuladora Pteris vittata L. encontrada em local onde o solo possuía $97 \mathrm{mg} \mathrm{kg}^{-1}$ de As (Ma, 2001). A primeira apresentou na parte aérea, $170 \mathrm{mg} \mathrm{kg}^{-1}$ de As, e na raiz, $1000 \mathrm{mg} \mathrm{kg}^{-1}(\mathrm{FA}=0,01)$, enquanto a $P$. vittata apresentou na parte aérea, $7234 \mathrm{mg} \mathrm{kg}^{-1}$ de As, e na raiz, $303 \mathrm{mg} \mathrm{kg}^{-1}(\mathrm{FA}=74,6)$.
Avaliando-se os dados referentes ao fator de bioacumulação (FA) da Brachiaria decumbens, é possível inferir que a espécie é tolerante ao As e não hiperacumuladora, uma vez que, apenas no solo LAd, nas doses de 50 e $200 \mathrm{mg} \mathrm{kg}^{-1}$ obteve-se FA >1. Contudo, comparando os valores de FA nessa classe de solo, nessas concentrações, com o FA da espécie hiperacumuladora $P$. vittata, não é possível considerar que houve hiperacumulação do elemento, uma vez que tais valores foram próximos de 1 , sendo 1,55 para a dose de $50 \mathrm{mg} \mathrm{kg}^{-1}$ de As e 1,04 para a dose de $200 \mathrm{mg} \mathrm{kg}^{-1}$ de As. Os valores referentes ao fator de FA e ao índice de translocação (IT) da braquiária são apresentados nas Figuras 5 e 6.

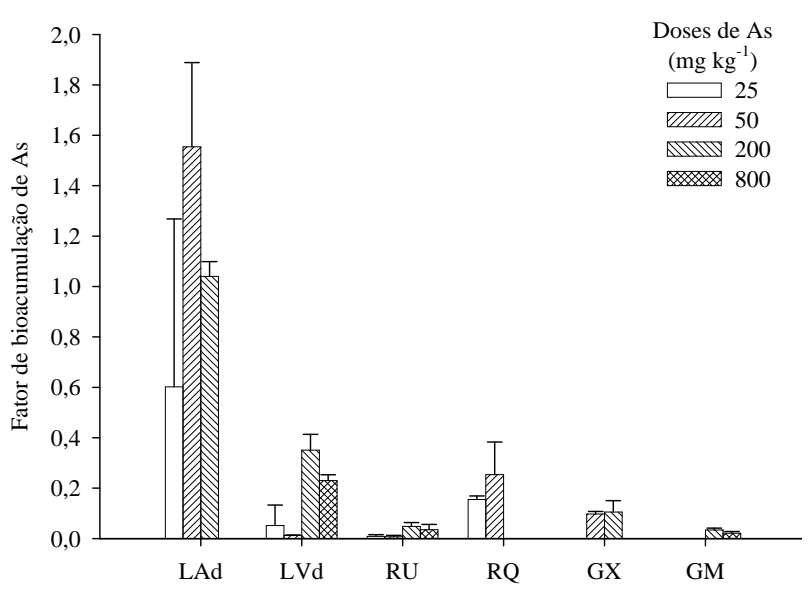

Figura 5 - Valores médios referentes ao fator de bioacumulação de As da Brachiaria decumbens cultivada em solos contaminados artificialmente com As.

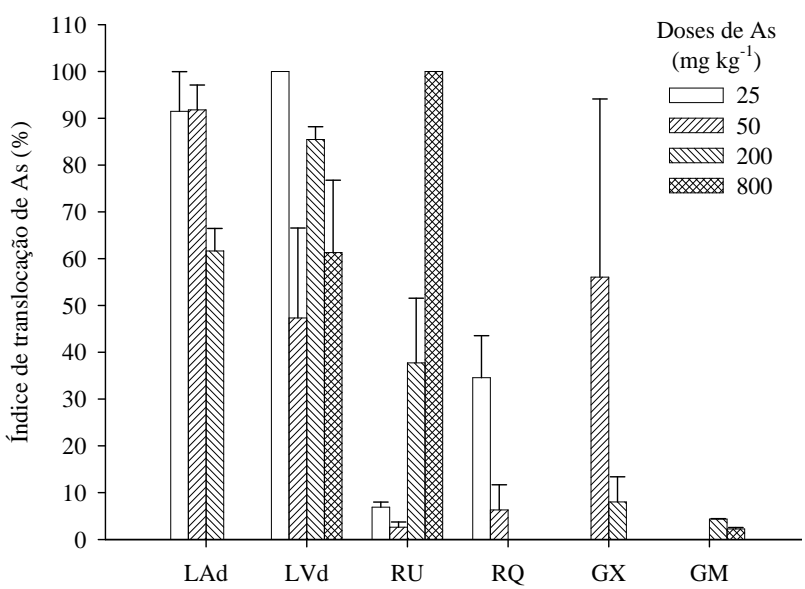

Figura 6 - Valores médios referentes ao índice de translocação de As (\%) da Brachiaria decumbens cultivada em solos contaminados artificialmente com As. 
Segundo Marin et al. (1993), o As tende a ser pouco translocado para a parte aérea das plantas após a absorção pelas raízes. Tal afirmação corrobora os resultados obtidos, uma vez que a translocação de As da raiz para a parte aérea da braquiária foi bastante restrita. Nota-se, de modo geral, que os maiores IT foram obtidos no solo LAd e LVd, enquanto, nos demais solos, o As ficou predominantemente retido na raiz.

\section{CONCLUSÕES}

Houve redução no desenvolvimento da braquiária de forma diferenciada nas seis classes de solos utilizadas.

A Brachiaria decumbens Stapf. é uma espécie que pode ser utilizada em programas de recuperação/ revegetação de áreas degradadas e contaminadas com arsênio.

\section{REFERÊNCIAS BIBLIOGRÁFICAS}

ABICHEQUER, A.D.; BOHNEN, H. Eficiência de absorção, translocação e utilização de fósforo por variedades de trigo. Revista Brasileira de Ciência do Solo, Viçosa, v.22, n.1, p.21-26, jan./mar. 1998.

AGENCY FOR TOXIC SUBSTANCES \& DISEASE REGISTRY. Priority list of hazardous substances. 2005. Disponível em: <http://www.atsdr.cdc.gov/cercla/ '0 $\overline{5}$ list.htmpl>. Acesso em: 15 set. 2006.

BAIRD, C. Química ambiental. 2.ed. Porto Alegre: Bookman, 2002. 621p.

BARRA, C.M.; SANTELLI, R.E.; ABRÃO, J.J.; GUARDIA, M. Especiação de arsênio - uma revisão. Química Nova, São Paulo, v.23, p.58-70, 2000.

BASU, A.; MAHATA, J.; GUPTA, S.; GIRI, A.R. Genetic toxicology of a paradoxical human carcinogen, arsenic: a review. Mutation Research, v.488, p. 171-194, 2001.

BORBA, R.P.; FIGUEIREDO, B.R.; CAVALCANTI, J.A. Arsênio na água subterrânea em Ouro Preto e Mariana, Quadrilátero Ferrífero (MG). Revista Esc. Minas, v. 57, p. 45-51, 2004.

BRASIL. Ministério da Agricultura e Reforma Agrária. Regras para análise de sementes. Brasília: CLAV/DNDV; SNAD/MA, 1992. 365p.
CAI, Y.; SU, J.; MA, L.Q. Low molecular weight thiols in arsenic hyperaccumulator Pteris vittata upon exposure to arsenic and other trace elements. Environmental Pollution, v.129, p.69-78, 2004.

CAILLE, N.; ZHAO, F.J.; MCGRATH, S.P. Comparison of root absorption, translocation and tolerance of arsenic in the hyperaccumulator Pteris vittata and the nonhyperaccumulartor Pteris tremula. New Phytologist, Palo Alto, v.165, p.755-761, 2005.

CAMPOS, M.L.; GUILHERME, L.R.G.; VISIOLLI, E.; ANTUNES, A.S.; CURI, N.; MARQUES, J.J.; SILVA, M.L.N. Força iônica da solução de equilíbrio na adsorção de arsênio em latossolos brasileiros. Pesquisa Agropecuária Brasileira, Brasília, v.41, n.3, p.457-460, 2006.

FAYIGA, A.O.; MA, L.Q.; CAO, X.; RATHINASABAPATHI, B. Effects of heavy metals on growth and arsenic accumulation in the arsenic hyperaccumulator Pteris vittata L. Environmental Pollution, v.132, p.289-296, 2004.

FERNANDES, L. A.; FAQUION, V.; FURTINI Neto, A. E.; CURI, N. Frações de fósforo e atividade da fosfatase ácida em plantas de feijoeiro cultivadas em solos de várzea. Revista Brasileira de Ciência do Solo, v. 24 561571,2000

FITZ, W.J.; WENZEL, W.W. Arsenic transformations in soil-rhizosphere-plant system: fundamentals and potential aplication to phytoremediation. Journal of Biotechnology, v.99, p.259-278, 2002.

GOH, K.H.; LIM, T.T. Geochemistry of inorganic arsenic and selenium in tropical soil: effect of reaction time, $\mathrm{pH}$ and competitive anions on arsenic and selenium adsortion. Chemosphere, v.55, p.849-859, 2004.

GONZAGA, M.I.S.; SANTOS, J.A.G.; MA, L.Q. Arsenic phytoextraction and hyperaccumulation by fern species. Science Agricola, Piracicaba, v.63, n.1, p.90101, 2006.

KOE, T. de; JAQUES, N.M.M. Arsenate tolerance in Agrostis castellana and Agrostis delicatula. Plant and Soil, Netherlands, v.151, p.185-191, 1993. 
LINDBLOM, S.D.; ABDEL-GHANY, S.; HANSON, B.R.; HWANG, S.; TERRY, N.; PILON-SMITS, E.A.H.

Constitutive expression of a high-affinity sulfate transporter in Indian mustard affects metal tolerance and accumulation. Journal Environmental Quality, v.35, p.726-733, 2006.

MA, L. A fern that hyperaccumulates arsenic. Nature, London, v.411, p.579, 2001.

MAGALHÃES, V.F.; PFEIFFER, W.C. Arsenic concentration in sediments near a metallurgical plant (Sepetiba Bay, Rio de Janeiro, Brazil). Journal Geochemistry Exploration, v.52, p.175-181, 1995.

MAGALHÃES, V.F.; CARVALHO, C.E.V.; PFEIFFER, W.C. Arsenic contamination and dispersion in the Engenho Inlet, Sepetiba Bay, SE, Brazil. Water, Air \& Soil Pollution, v.129, p.83-90, 2001.

MANDAL, B.K.; SUZUKI, K.T. Arsenic around the world: a review. Talanta, Oxford, v.58, p.201-235, 2002.

MARIN, A.R.; MASSCHELEYN, P.H.; PATRICK

JUNIOR, W.H. Soil redox-pH stability of arsenic species and its influence on arsenic uptake by rice. Plant and Soil, Netherlands, v.152, p.245-253, 1993.

MATSCHULLAT, J.; BORBA, R.P.; DESCHAMPS, E.; FIGUEIREDO, B.R.; GABRIO, T.; SCHWENK, M. Human and environmental contamination in the Iron Quadrangle, Brazil. Applied Geochemistry, v.15, p.193-202, 2000.

RODRIGUEZ, R.R.; BASTA, N.T.; CASTEEL, S.W.; ARMSTRONG, F.P.; WARD, D.C. Chemical extraction methods to assess bioavailable arsenic in soil and solid media. Journal Environment Quality, v.32, p.876-884, 2003.

SKOOG, D.A.; HOLLER, F.J.; NIEMAN, T.A. Princípios de análise instrumental. 5.ed. Porto Alegre: Bookman, 2002. 836p.

SMITH, E.; NAIDU, R.; ALSTON, A.M. Chemistry of inorganic arsenic in soils: II., effect of phosphorus, sodium, and calcium on arsenic sorption. Journal of Environmental Quality, v.31, p.557-563, 2002.

SOUZA, R. F.; FAQUIN, V.; TORRES, P. R. F.; BALIZA, D. P. Calagem e adubação orgânica: Influência na adsorção de fósforo em solos. Revista Brasileira de Ciência do Solo, v. 30, 975-983, 2006. 